\title{
Composição e variabilidade espaço-temporal da meiofauna de uma praia arenosa na região amazônica (Ajuruteua, Pará)
}

\author{
Tatianne P. Gomes \& José S. Rosa Filho
}

Laboratório de Oceanografia Biológica, Faculdade de Oceanografia, IG, Universidade Federal do Pará, Avenida Augusto Côrrea, 1, 66075110 Belém, PA, Brasil. (tatiannepgomes@yahoo.com.br; jsouto@ufpa.br)

\begin{abstract}
Composition and spatio-temporal variability of meiofauna community on a sandy beach in the Amazon region (Ajuruteua, Pará, Brazil). The present study investigates the spatio-temporal changes in the meiofauna community along the intertidal zone of Ajuruteua Beach, State of Pará, Brazil. Samples were collected during spring tides at different levels of the beach face every two months from April 2003 to February 2004. The material was sampled using a cylindrical corer $\left(3.14 \mathrm{~cm}^{2}\right)$ and fixed with 5\% saline formalin. In the laboratory, samples were sieved through a $0.063 \mathrm{~mm}$ mesh screen and the retained organisms were identified at higher taxonomic levels, counted and preserved in ethanol 70\%. Meiofauna was composed of eight groups: Turbellaria, Nematoda, Tardigrada, Polychaeta, Oligochaeta, Acari, Copepoda and nauplius (Copepoda). Nematoda was the dominant group, representing $74 \%$ of total organisms, followed by Copepoda (19\%). A clear faunal zonation was observed on three lines parallel to the shoreline, which showed significant differences in abundance, richness and density of the main taxonomic groups. The mean density was lowest in April and highest in December. The highest densities and richness were recorded in the mid intertidal zone, while the lowest values were recorded in the upper and lower intertidal zones. Meiofauna community was most rich and abundant during the dry months, although differences were not significant between climatic periods. The main factors involved in the spatio-temporal variations of meiofauna were wave and tidal action and salinity fluctuations.
\end{abstract}

KEYWORDS. Benthos, meiofauna, community ecology, vertical zonation, Amazon.

RESUMO. Este trabalho avalia a variabilidade espaço-temporal da meiofauna do médiolitoral na praia de Ajuruteua, Estado do Pará. As coletas foram realizadas a cada dois meses, entre abril de 2003 a fevereiro de 2004 durante as marés de sizígia, em diferentes zonas da praia. As amostras foram retiradas com um amostrador cilíndrico de $3,14 \mathrm{~cm}^{2}$ e fixadas em formalina salina a $5 \%$. Em laboratório, as amostras foram passadas em malha de $0,063 \mathrm{~mm}$ de abertura e os organismos retidos identificados em nível de grandes grupos taxonômicos, contados e fixados em álcool etílico a $70 \%$. A meiofauna esteve representada por oito grupos: Turbellaria, Nematoda, Tardigrada, Polychaeta, Oligochaeta, Acari, adultos de Copepoda Harpacticoida e juvenis de Copepoda Harpacticoida. Nematoda foi o grupo dominante, representando $74 \%$ do total de indivíduos, seguido de Copepoda $(19 \%)$. Pôde-se observar clara zonação horizontal da fauna, que se distribuiu em três faixas paralelas à linha de praia, com características significativamente distintas quanto à abundância, riqueza e densidade dos principais grupos taxonômicos. No médiolitoral médio foram observados valores significativamente mais elevados de riqueza e abundância, enquanto os valores mais baixos foram registrados no médiolitoral superior e inferior. A comunidade de meiofauna, ainda que não tenha variado significativamente entre períodos climáticos, foi mais rica e abundante nos meses secos. Os principais fatores responsáveis pelas variações espaço-temporais da meiofauna foram a ação das ondas e das marés e as variações na salinidade da água.

PALAVRAS-CHAVE. Bentos, meiofauna, distribuição espaço-temporal, Amazônia.

As praias arenosas oceânicas constituem sistemas de elevada instabilidade ambiental sujeitos a bruscas variações energéticas geradas por processos eólicos, biológicos e hidráulicos. Devido à interação desses fatores, as praias sofrem mudanças morfológicas e trocas de sedimento com regiões adjacentes em diferentes escalas temporais (SHORT, 2003). Como conseqüência deste presumido rigor ambiental, estas áreas foram muitas vezes consideradas desertos biológicos (MCLACHLAN \& BROWN, 2006), e somente em anos recentes foi demonstrado que sua fauna bentônica pode ser diversa e abundante (ARMONIES \& REISE, 2000; RodRíGUEZ, 2004; McLachlan \& DoRvlo, 2005).

A meiofauna, composta por metazoários com dimensões entre $0,044 \mathrm{~mm}$ a $0,5 \mathrm{~mm}$, é uma das mais importantes associações biológicas de praias arenosas. Integram a dieta alimentar de diversas espécies, de macroinvertebrados a peixes, e participam nos processos de biomineralização da matéria orgânica (Coull, 1988). Nestes ambientes, a meiofauna é composta por um grande número de táxons, principalmente Nematoda, Copepoda, Oligochaeta, Turbellaria, Gastrotricha, Kinorhyncha,
Gnatostomulida e Tardigrada (RAfaelli \& Hawkins, 1996), presentes em densidades médias que variam de $1.000 \mathrm{a}$ 2.000 ind. $10 \mathrm{~cm}^{-2}$ (McLachlan \& BRown, 2006).

Em ambientes praiais, a distribuição da meiofauna é condicionada por diferentes escalas espaciais e temporais. Espacialmente, observa-se grande agregação horizontal e vertical, sendo a granulometria do sedimento, salinidade da água, tensão de oxigênio, composição química da água intersticial e disponibilidade de alimento citadas como as principais características ambientais geradoras desse padrão (GIERE, 1993; NDARO et al., 1995). A variabilidade temporal da meiofauna se dá em pequenas (relacionada ao ciclo das marés e mudanças na umidade no sedimento), médias (base diária, relacionada às mudanças de temperatura) e longas escalas (ligada às estações do ano) (McLachlan \& Brown, 2006). Além das características ambientais, adicionam complexidade à variabilidade espaço-temporal da meiofauna em praias arenosas, às interações biológicas e a história de vida das espécies (GIERE, 1993; KAISER et al., 2005).

Apesar de um razoável número de estudos sobre a meiofauna de praias arenosas em diversas regiões do 
planeta (McLaChlan, 1980; CALles et al., 2002; KotwiCKI et al., 2005b; Harriague et al., 2006), as pesquisas em praias arenosas tropicais e polares são ainda incipientes, quando comparadas com outras zonas climáticas (GIERE, 1993). No Brasil, os estudos sobre a meiofauna em praias arenosas limitam-se aos estados do Rio Grande do Norte, Pernambuco, Rio de Janeiro, São Paulo e Santa Catarina, podendo ser citando os estudos de MedeIros (1984), Esteves et al. (1998), Bezerra et al. (1996, 1997), Wandeness et al. (1997), NetTo et al. (1999), NogueIRA \& Skowronski (2001) e Pinto \& SAntos (2006).

Pouco se sabe a respeito do grupo na Região Norte do Brasil, com exceção dos estudos de PAUlA et al. (2006) e Rosa Filho et al. (2007), que avaliaram os impactos da carcinicultura sobre a meiofauna no estuário de Curuçá (PA), ainda que esta seja identificada por CAPOBIANCO et al. (2001) e MMA (2007) como prioritária para a conservação da biodiversidade de invertebrados bentônicos costeiros.

O presente estudo teve como objetivo descrever as variações espaço-temporais na composição taxonômica, densidade e riqueza de táxons da meiofauna no médiolitoral da praia arenosa de macromaré de Ajuruteua (Bragança, PA).

\section{MATERIALEMÉTODOS}

Área de estudo. A praia de Ajuruteua (Fig.1), localizada na região nordeste do estado do Pará, apresenta extensa área plana orientada na direção NW-SE, de aproximadamente 2,5 km de extensão e $300 \mathrm{~m}$ de largura. Situa-se entre o Canal da Barca, que a separa das praias dos pescadores, e o Canal do Chavascal, limite com as praias do Farol Velho e Chavascal (SouzA Filho et al., 2004). Segundo Alves \& El-Robrini (2006) e Lima et al.

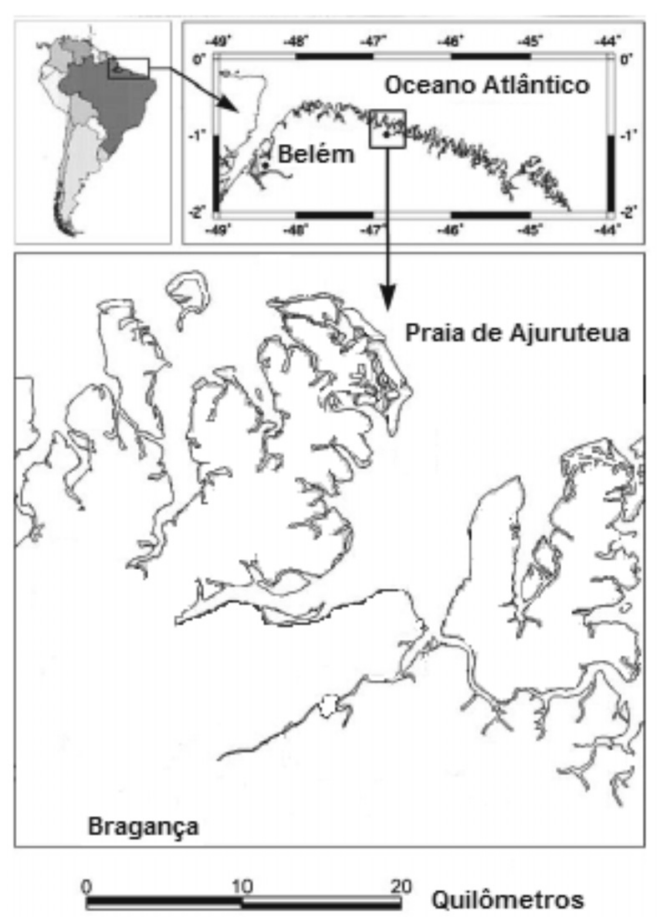

Figura 1. Mapa de localização da área de estudo (modificado de Krause \& SoARES, 2004).
(2007), a praia apresenta baixa declividade (média de $\left.1^{\circ} 52^{\prime}\right)$, arrebentação do tipo deslizante e sedimento composto por areia fina (diâmetro médio dos grãos variando entre 2,55 e $3 \phi$ ). Tais características fazem com que Ajuruteua seja classificada como dissipativa, com valores do parâmetro de Dean $(\Omega)$ variando entre 6,95 e 8,3 nos períodos seco e chuvoso, respectivamente.

Souza Filho et al. (2003) subdividiram a praia de Ajuruteua em três zonas: 1. Supralitoral, localizada entre a linha de maré alta de sizígia e a base das dunas; 2. Médiolitoral, compreendida entre as linhas de maré alta e baixa de sizígia, podendo ser subdividida em médiolitoral superior, médio e inferior, com extensão de aproximadamente $260 \mathrm{~m}$; e 3 . Infralitoral, abaixo da linha de maré baixa de sizígia.

O clima na região é do tipo Am2 (segundo adaptação da classificação de Köppen), caracterizado por ser quente e úmido (temperatura acima dos $23^{\circ} \mathrm{C}$ ao longo de todo o ano) (MArtorrano et al., 1993), com estação seca prolongando-se de julho a dezembro, e um período chuvoso bem acentuado, com fortes chuvas nos demais períodos do ano (janeiro a junho). A pluviosidade média anual varia de 2.300 a $2.800 \mathrm{~mm}$ e a umidade relativa do ar entre 80 e $91 \%$ (MorAes et al., 2005).

Procedimentos de campo e laboratório. As coletas foram realizadas no médiolitoral, com periodicidade bimestral, entre abril de 2003 e fevereiro de 2004, durante marés de sizígia. O desenho amostral foi do tipo estratificado ao longo de quatro transectos perpendiculares à linha de costa. Para a definição dos estratos, utilizou-se a divisão da zona entremarés proposta por Souza Filho et al. (2003), com coleta de amostras no entremarés superior (EMS), médio (EMM) e inferior (EMI) (Fig. 2). Em cada estrato foram coletadas quatro unidades amostrais, utilizando amostrador cilíndrico de $3,14 \mathrm{~cm}^{2}$ de área, enterrado a $10 \mathrm{~cm}$ de profundidade no sedimento. As amostras foram fixadas em formalina salina a 5\%, corada com Rosa de Bengala.

Em laboratório, as amostras biológicas foram separadas do sedimento por elutriação manual, técnica que consta de centrifugações manuais em Becker de $1 \mathrm{~L}$ e sucessivas lavagens sob água corrente, sendo o sobrenadante passado em uma peneira com malha de $0,063 \mathrm{~mm}$ de abertura. Esse procedimento foi repetido cinco vezes para cada unidade de amostra, para maior eficiência. Os organismos retidos foram identificados em nível de grandes grupos, com o auxílio de microscópio estereoscópico, armazenados e conservados em álcool etílico a $70 \%$.

Dados de temperatura do ar e precipitação pluviométrica foram registrados na Estação Meteorológica de Bragança (DM/UFPA), situada no manguezal próximo à praia. Os valores de salinidade da água foram registrados em cada ocasião de coleta, com o auxílio de um refratômetro manual marca Atago. As coletas e caracterização dos sedimentos foram realizadas pela equipe de sedimentologia do Projeto Milênio "Uso e Apropriações de Recursos Costeiros/RECOS (CNPq/ PADCT/ MCT)".

Para comparação dos descritores (densidade e riqueza) entre os estratos e os períodos de coletas, foi utilizada análise de variância (ANOVA) bi-fatorial. Dado 


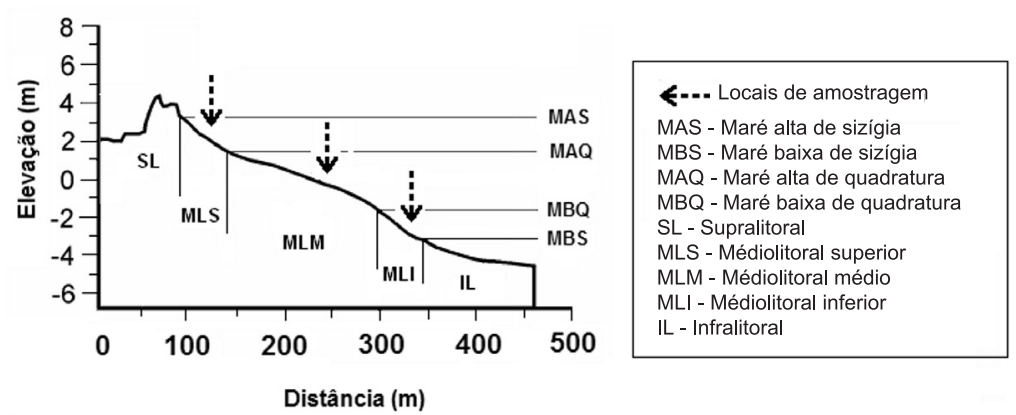

Figura 2. Esquema de amostragem da macrofauna bentônica utilizado na praia de Ajuruteua, Pará (adaptado de SouzA FiLHo et al., 2003).

que ao comparar os descritores entre transectos em cada ocasião de amostragem, utilizando ANOVA unifatorial, não foram identificadas diferenças significativas, os dados dos quatro transectos foram agrupados para as análises posteriores. Antes da realização destas análises, os dados foram testados quanto à normalidade da distribuição (Teste de Kolmogorov-Smirnov) e homogeneidade das variâncias (Teste de Levene), sendo transformados para $\log (\mathrm{x}+1)$ quando necessário.

Para descrever as principais tendências de variabilidade da meiofauna local, utilizou-se uma análise de escalonamento multidimensional (MDS), baseada em matriz de similaridade calculada utilizando o índice de similaridade de Bray-Curtis, com os dados de abundância transformados para raiz quarta. Para avaliar a significância dos estratos e períodos na introdução de variabilidade biológica utilizou-se uma Análise de Similaridade (ANOSIM) (Clarke \& WarWick, 2001).

\section{RESULTADOS}

A temperatura do ar variou entre $26^{\circ} \mathrm{Ce} 28^{\circ} \mathrm{C}$, sendo máxima em dezembro de 2003 e mínima em fevereiro de 2004. Os meses de janeiro a maio foram os mais chuvosos e os menores valores de precipitação pluviométrica foram registrados entre agosto e dezembro. Acompanhando a precipitação, porém com comportamento inverso, a salinidade da água foi mínima em abril e máxima em dezembro (Fig. 3).

As características dos sedimentos praticamente não variaram entre estratos e ocasiões de amostragem. O diâmetro médio dos grãos esteve entre 2,6-2,9 Ø, com os menores valores sendo registrados no período chuvoso; o

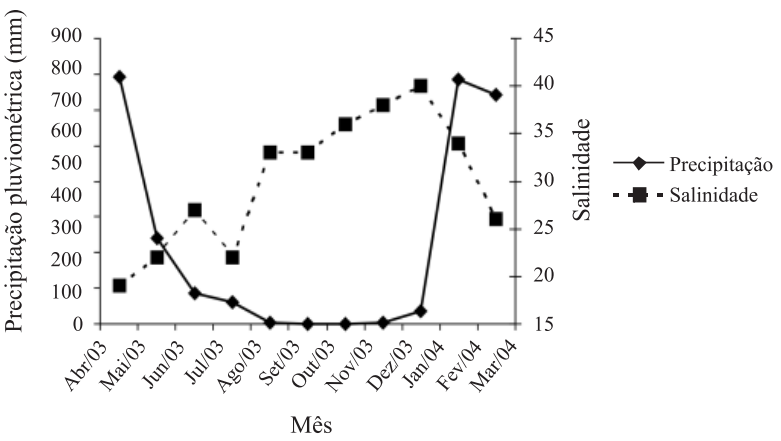

Figura 3. Taxa de precipitação pluviométrica e salinidade da água na praia de Ajuruteua, Pará, entre abril de 2003 e fevereiro de 2004. grau de seleção oscilou entre muito bem selecionado e moderadamente selecionado, com o desvio padrão oscilando de 0,34 a 0,57 ; a assimetria variou de simétrica a positiva ($0,07$ a 0,28$)$, havendo tendência à assimetria negativa nos períodos de maiores precipitações. A curtose variou de 1,06 a 1,19 , sendo as distribuições predominantemente leptocúrticas (Braga \& Souza Filho, 2007).

A meiofauna esteve composta por oito grupos: Turbellaria, Nematoda, Tardigrada, Polychaeta, Oligochaeta, Acarina, Copepoda Harpacticoida adultos e Copepoda Harpacticoida juvenis, estando sete presentes no período seco e oito no chuvoso. Merece destaque o fato de que no mês de abril, período chuvoso, foram registrados apenas Nematoda e Copepoda.

A abundância relativa dos grupos foi semelhante ao longo do ano, com um pequeno aumento na participação de Copepoda no período chuvoso. Os demais grupos foram pouco abundantes, representando sempre menos do que $1 \%$ do total de indivíduos. Em ambos os períodos, os Nematoda dominaram em todas as zonas da praia, sendo as maiores abundâncias do grupo registradas no médiolitoral superior, onde estes representaram sempre mais de $94 \%$ do total de indivíduos. No médiolitoral médio observou-se aumento da importância dos copépodos, que representaram $25 \%$ e $31 \%$ dos organismos nos períodos seco e chuvoso respectivamente (Tab. I).

Tabela I. Participação relativa dos grupos da meiofauna por estratos durante o período seco e chuvoso na praia de Ajuruteua, Pará. (EMI, entremarés inferior; EMM, entremarés médio; EMS, entremarés superior).

\begin{tabular}{lrcr}
\hline Grupos & \multicolumn{3}{c}{ Período seco (\%) } \\
\cline { 2 - 4 } Nematoda & EMS & EMM & EMI \\
Copepoda & 94,39 & 59,86 & 94,86 \\
Turbellaria & 0,29 & 24,97 & 0,75 \\
Tardigrada & 1,28 & 19,65 & 2,59 \\
Nauplius & 3,63 & 4,04 & 1,31 \\
Acari & 0,00 & 0,16 & 0,19 \\
Polychaeta & 0,12 & 0,03 & 0,00 \\
Oligochaeta & 0,29 & 0,32 & 0,29 \\
Grupos & 0,00 & 0,00 & 0,00 \\
\hline Nematoda & & Período chuvoso $\%)$ & \\
Copepoda & 96,62 & EMM & EMI \\
Turbellaria & 1,09 & 65,51 & 95,62 \\
Tardigrada & 0,41 & 30,38 & 2,19 \\
Nauplius & 1,27 & 1,88 & 0,90 \\
Acari & 0,13 & 3,34 & 2,16 \\
Polychaeta & 1,58 & 0,80 & 0,04 \\
Oligochaeta & 0,00 & 0,04 & 0,05 \\
\hline & 0,09 & 0,10 & 0,11 \\
& & 0,03 & 0,00 \\
\hline
\end{tabular}


As riquezas e densidades foram mais elevadas no período seco $(2,97 \pm 1,28$ grupos e $673,92 \pm 553,42$ ind. 10 $\left.\mathrm{cm}^{-2}\right)$ do que no chuvoso $(2,83 \pm 1,48$ grupos e $617,85 \pm 552,71$ ind. $10 \mathrm{~cm}^{-2}$ ), embora a diferença não tenha sido significativa. Nos meses secos, as maiores riquezas e densidades ocorreram em junho $(3,37 \pm 0,15$ grupos e $715,46 \pm 250$ ind. $\left.10 \mathrm{~cm}^{-2}\right)$ e as menores em agosto $(2,17 \pm 0,17$ grupos e $467,85 \pm 152,59$ ind. $\left.10 \mathrm{~cm}^{-2}\right)$. Na época chuvosa, a maior riqueza foi registrada em fevereiro $(3,57 \pm 0,17$ grupos) e a maior abundância em dezembro $(746,76 \pm 222$ ind. $10 \mathrm{~cm}^{-2}$ ); por sua vez, neste período os menores valores de riqueza e densidade foram registrados em abril $\left(1,46 \pm 0,07\right.$ grupos e $199,94 \pm 48,28$ ind. $\left.10 \mathrm{~cm}^{-2}\right)$ (Fig. 4).

A riqueza variou significativamente entre zonas da praia nos períodos seco e chuvoso. Em ambos os períodos, o maior número de táxons foi registrado no médiolitoral médio $(3,73 \pm 0,08$ e $3,73 \pm 0,15$ grupos nos períodos seco e chuvoso respectivamente) e o menor no médiolitoral superior $(1,81 \pm 0,16$ e 1,73 $\pm 0,13$ grupos nos períodos seco e chuvoso respectivamente) (Fig. 5).

Também a densidade variou significativamente entre as zonas nos dois períodos climáticos, sendo as maiores densidades observadas no médiolitoral médio (713,23 $\pm 36,65$ ind. $\left.10 \mathrm{~cm}^{-2}\right)$ no período seco e no médiolitoral inferior $\left(662,61 \pm 52,86\right.$ ind $\left.10 \mathrm{~cm}^{-2}\right)$ no chuvoso. Por sua vez, os menores valores de abundância foram registrados no médiolitoral inferior durante o período seco $\left(481,14 \pm 48,59\right.$ ind $\left.10 \mathrm{~cm}^{-2}\right)$ e no médiolitoral superior na estação chuvosa $\left(181,65 \pm 24,55\right.$ ind. $\left.10 \mathrm{~cm}^{-2}\right)$ (Fig. 5).

Nos períodos seco e chuvoso as amostras do médiolitoral médio separaram-se das demais (Fig. 6). Por sua vez, as amostras do médiolitoral superior e inferior estiveram sempre agrupadas. Ao comparar a estrutura das associações de meiofauna, foi identificada diferença significativa entre zonas da praia em ambos os períodos climáticos $(\mathrm{R}=0,56$ e $\mathrm{p}=0,001$ no período seco e $\mathrm{R}=0,324$ e $\mathrm{p}=0,001$ no período chuvoso) e entre os meses secos e chuvosos $(\mathrm{R}=0,053$ e $\mathrm{p}=0,001)$.

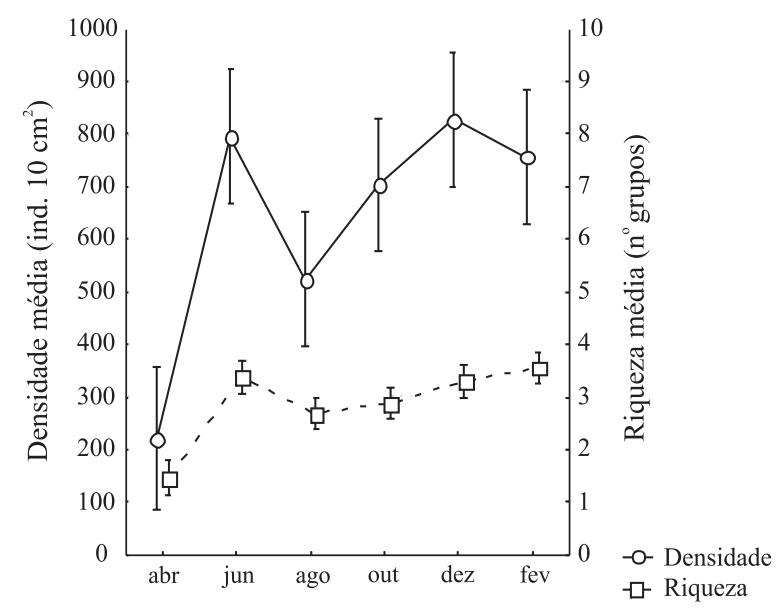

Figura 4. Valores médios de densidade e riqueza da meiofauna ao longo do ano no médiolitoral da praia de Ajuruteua, Pará. As barras verticais indicam o intervalo de confiança (95\%).

\section{DISCUSSÃO}

A composição taxonômica e o número de grupos da meiofauna registrados em Ajuruteua são semelhantes aos citados para outras praias arenosas (RAFAELLI \& Hawkins, 1996, Rodríguez et al., 2003; Pinto \& Santos, 2006). Também a elevada dominância de Nematoda, seguido de Copepoda, concorda com o padrão global de abundância relativa dos grandes grupos da meiofauna nos trópicos (Coull, 1988).

Kотшіскі et al. (2005a), ao estudarem os padrões mundiais de biodiversidade da meiofauna em praias arenosas, observaram que Nematoda é o grupo dominante em regiões quentes, representando em média $69,6 \%$ do total de organismos e sendo substituído por Turbellaria nos pólos. No presente estudo, os Nematoda perfizeram em média $84,5 \%$ dos indivíduos coletados. O elevado sucesso deste grupo em praias arenosas como Ajuruteua se deve, como apontado por Bouwman (1983), a três razões principais: alta tolerância a variados estressores ambientais, diversidade de estratégias alimentares e grande facilidade de enterramento no sedimento.

As densidades médias da meiofauna registradas em Ajuruteua $\left(646,47 \pm 553,05\right.$ ind. $\left.10 \mathrm{~cm}^{-2}\right)$ assemelham-se àquelas observadas por BEZERRA et al. (1996) e SOUZA-
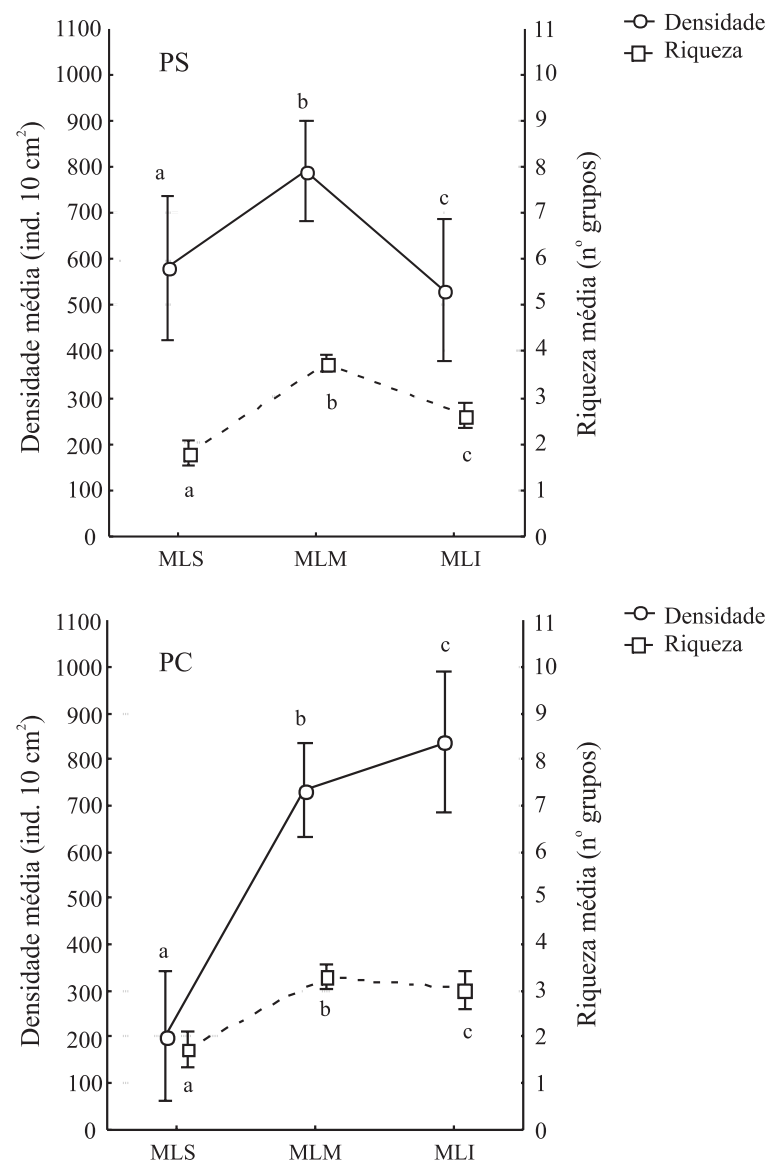

Figura 5. Valores de riqueza e densidade nos estratos estudados ao longo dos anos, no período seco (PS) e no período chuvoso (PC), na zona entremarés da praia de Ajuruteua, Bragança, Pará (MLI, médiolitoral inferior; MLM, médiolitoral médio; MLS, médiolitoral superior). As barras verticais indicam o erro padrão e as letras o resultado dos testes de contraste de médias. 

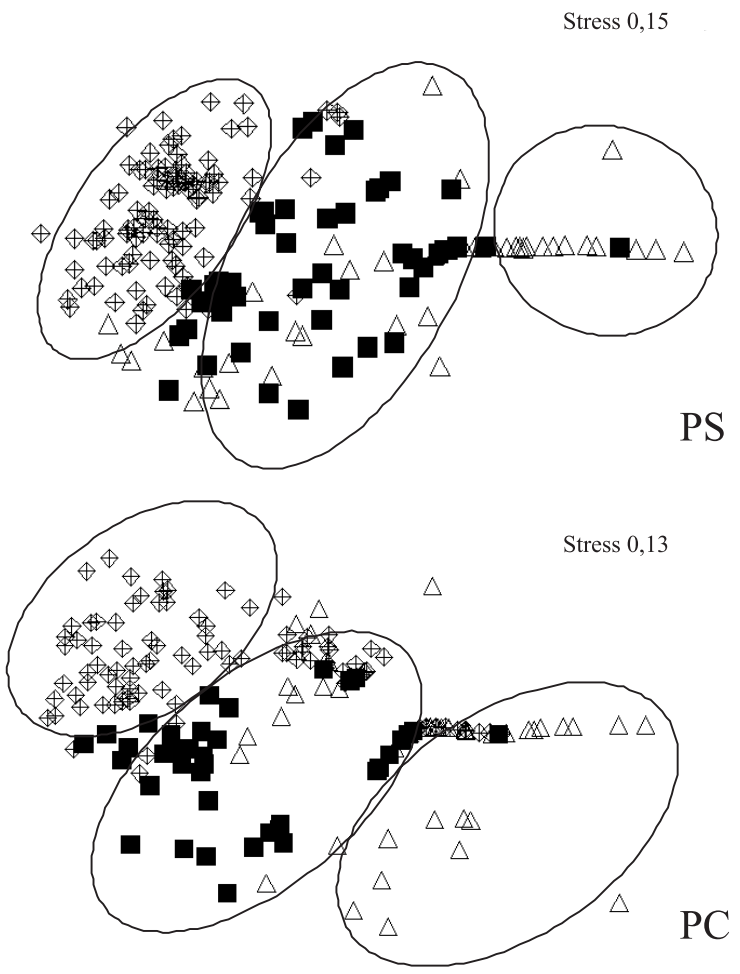

$\triangle \mathrm{MLS} \quad \oplus \mathrm{MLM} \quad \square \mathrm{MLI}$

Figura 6. Configuração da análise de escalonamento multidimensional (MDS) com os dados de abundância transformados para raiz quarta durante os períodos seco (PS) e chuvoso (PC) na zona entremarés da praia de Ajuruteua, Pará (MLI, médiolitoral inferior; MLM, médiolitoral médio; MLS, médiolitoral superior).

Santos et al. (2003) em praias arenosas de Pernambuco. KотіWICKI et al. (2005a) indicam serem esperadas em praias arenosas tropicais densidades de meiofauna da ordem de $538 \pm 137$ ind. $10 \mathrm{~cm}^{-2}$.

Em Ajuruteua observou-se clara zonação horizontal da meiofauna ao longo da face da praia, com a fauna distribuindo-se em três estratos distintos: superior, médio e inferior; correspondendo às zonas seca, de retenção e de ressurgência identificadas por MCLACHLAN (1980) em praias arenosas africanas. Segundo este autor, a distribuição diferencial da meiofauna em praias arenosas ocorre em resposta às variações na altura da maré e na ação das ondas. A zona seca tem temperatura muito variável e o conteúdo de água pode ficar abaixo de $50 \%$ na maré baixa; na zona de retenção, a temperatura é quase constante e a taxa de saturação de oxigênio está sempre acima de $50 \%$; por fim, a zona de retenção está permanentemente encharcada de água e a taxa de saturação de oxigênio varia entre 40 e 70\% (MCLACHLAN, 1980; MCLACHLAN \& TuRNer, 1994).

No presente estudo, as principais modificações da meiofauna ao longo da face da praia relacionaram-se à participação relativa dos grandes grupos taxonômicos, riqueza e abundância. Nas zonas de entremarés superior e inferior, Nematoda representou sempre mais que $90 \%$ do total de organismos, enquanto no meio da praia a participação relativa de Copepoda variou entre 25 e $30 \%$. Tal padrão deve-se, provavelmente, ao fato de que, com poucas exceções, os Copepoda são mais sensíveis à depleção de oxigênio ou anoxia que os Nematoda (Moodley et al., 2000). Isto explicaria, a elevada dominância de Nematoda nos extremos do médiolitoral, onde a disponibilidade de oxigênio é mínima, e o aumento da abundância de Copepoda no médiolitoral médio onde os interstícios estão saturados de oxigênio.

De uma forma geral, valores significativamente mais elevados de riqueza e abundância foram observados no médiolitoral médio, que corresponde à zona úmida de McLachlan (1980). McLachlan (1983) e McLachlan \& TURNER (1994) citam que esta zona é a mais adequada à existência de ricas e densas populações de meiofauna, dado que apresenta as condições mais favoráveis para a vida de organismos intersticiais quanto ao balanço entre conteúdo de água, aporte de oxigênio e estabilidade física. PENNAK (1951) aponta ainda que nesta zona está presente uma microfauna mais rica e diversa que nos extremos, o que aumenta a disponibilidade de alimento para os organismos da meiofauna.

As características dos sedimentos têm sido citadas como dos fatores mais importantes para o estabelecimento de comunidades bentônicas marinhas (Thorson, 1957; Snelglove \& Buttman, 1994; Pinto \& SANTOS, 2006). Em Ajuruteua, no entanto, dado que ao longo do ano em toda a face da praia o sedimento dominante é areia fina bem selecionada (Krause \& SoARes, 2004; Alves \& El-Robrini, 2006; Lima et al., 2007), é pouco provável que a textura dos sedimentos tenha desempenhado papel de grande importância na estruturação da meiofauna. Modificações na estrutura das comunidades de meiofauna relacionadas a mudanças na granulometria dos sedimentos seriam esperadas caso houvesse variações marcantes no tamanho médio dos grãos (McLACHLAN et al., 1981; 'OlAFSSON, 1991), como se observa ao passar de praias dissipativas para reflectivas (SHORT, 2003; RODRíGUEZ et al., 2003).

Temporalmente, ainda que não tenham sido registradas modificações significativas na estrutura da comunidade de meiofauna, observou-se maior riqueza e densidade no período seco. Souza-Santos et al. (2003) citam que em Tamandaré (PE), a densidade de meiofauna foi menor no período chuvoso do que no seco devido ao efeito combinado da baixa salinidade e da erosão dos sedimentos superficiais nos meses de chuva. Segundo Pollok (1970) o aporte de água doce em praias não favorece os organismos da meiofauna, uma vez que além de reduzir a salinidade intersticial, remove dos sedimentos algumas populações microbianas essenciais para alimentação de organismos meiofaunais.

Concordando com os autores citados, no presente estudo a redução da salinidade e o aumento da hidrodinâmica nos meses chuvosos foram os principais fatores estruturadores da meiofauna. Na região do Salgado paraense, onde está a praia de Ajuruteua, entre janeiro e junho ocorrem as máximas precipitações pluviométricas, podendo ser registrados até $1000 \mathrm{~mm} / \mathrm{mês}$ de chuvas (Moraes et al., 2005). Tal abundância de chuvas provoca bruscas quedas da salinidade, acarretando restrição ou exclusão de grupos faunísticos exclusivamente marinhos ou pouco adaptados à baixa salinidade (KINNE, 1971). Esse efeito é particularmente confirmado em Ajuruteua em abril, 
mês com maior precipitação e menor salinidade, quando foi registrada a menor riqueza, com a presença apenas de Nematoda e Copepoda.

Segundo Wright \& SHORT (1984), em geral as ondas atingem maiores alturas e as ressacas são mais frequentes no período chuvoso, ocasionando erosão na zona entremarés. Nestas ocasiões, segundo RodRíGUEZ (2004), em praias arenosas com sedimentos finos a meiofauna está exposta à ação erosiva das ondas, que causa a suspensão de grande número de organismos para a coluna d'água, aumentando as taxas de mortalidade. Processos similares parecem ocorrer em Ajuruteua, onde o médiolitoral é erodido com o aumento da energia das ondas e das correntes de marés no período chuvoso, acarretando o aplainamento da face da praia, com grande mobilização de sedimentos para o infralitoral (Alves \& EL-RobRINI, 2006).

Agradecimentos. Ao Projeto "Uso e Apropriações de Recursos Costeiros/Milênio RECOS (CNPq/ PADCT/ MCT)", Grupo Temático: Modelagem, Manejo, Erosão e Ocupação Costeira (MM\&OC), no âmbito do qual foi realizado este estudo. Ao Projeto "Large Scale Biosphere-Atmosphere Experience in Amazonia (CNPq/PADCT/MCT)" pela cessão dos dados meteorológicos. Ao Conselho Nacional de Desenvolvimento Científico e Tecnológico (CNPq), pela concessão da bolsa Iniciação Científica à primeira autora durante a realização deste trabalho. A Profa. Dra. Taciana Kramer de Oliveira Pinto e ao Prof. Dr. Paulo da Cunha Lana pelas sugestões ao manuscrito. Ao técnico Afonso Quaresma, sem a ajuda do qual as coletas de campo não teriam sido possíveis.

\section{REFERÊNCIAS BIBLIOGRÁFICAS}

Alves, M. A. M. S. \& El-Robrini, M. 2006. Morphodynamics of a macrotidal beach: Ajuruteua, Bragança, North Brazil. Journal of Coastal Research SI(39):1848-1850.

Armonies, W. \& Reise, K. 2000. Faunal diversity across a sandy shore. Marine Ecology Progress Series 196:49-57.

Bezerra, T. N. C.; FonsêCa-Genevois, V. \& Genevois, B. 1996. Distribuição horizontal e vertical da meiofauna em uma região tropical intermareal (Istmo de Olinda-Pernambuco-Brasil). Trabalhos Oceanográficos da UFPE 24:249-262.

Bezerra, T. N. C; Genevois, B. \& FonsêCa-Genevois, V. 1997. Influência da granulometria na distribuição e adaptação da meiofauna na praia arenosa do Istmo de Olinda - PE. Oecologia Brasiliensis 3:107-116.

Boumman, L. A. 1983. A survey of nematodes from the Sem estuary. Part II. Species assemblages and associations. Zoological Journal of Systematics 110:345-376.

Braga, F. P. S. \& Souza-Filho, P. W. M. 2007. Sedimentologia e morfologia da praia de macromaré de Ajuruteua, Pará: um estudo para a definição de índices de sensibilidade ambiental ao derramamento de óleo. In: 40 PDPETRO, Campinas, Anais... Campinas, Brasil, 2007. p.19-27.

Calles, A. K.; Dominguéz, L.; Guartatanga, S.; Ruiz, V.; GonzÁlez, K.; Grunauer, M. P. C. R \& Vincx, M. 2002. Interannual variability of the meiobenthos communities from two Ecuadorian sandy beaches (1999-2001). Investigaciones Marinas 30(1): $1-5$

Capobiano, J. P. R.; Veríssimo, A.; Moreira, A.; Sawyer, D.; Santos, I. \& Pinto, L. P. 2001. Biodiversidade da Amazônia brasileira: avaliação de áreas prioritárias para a conservação, uso sustentável e repartição de benefícios. São Paulo, Instituto Socioambiental. 540p.

Clarke, K. R. \& Warwick, R. M. 2001. Changes in marine communities: an approach to statistical analysis and interpretation. $2^{\text {nd }}$ ed. Plymouth, Plymouth Marine Laboratory. $144 \mathrm{p}$

Coenn, J. C. P.; Silva Dias, M. A. F. \& Nobre, C. A. 1989. Aspectos climatológicos das linhas de instabilidade na Amazônia. Climanálise 4(11):34-40.
Coull, B. C. 1988. The ecology of the marine meiofauna. In: Higgins, R. P. \& Thiel, H. eds. Introduction to the Study of Meiofauna. Washington, Smithsonian Institute. p.18-38.

Esteves, A. M.; Bloise, C. \& Nogueira, C. S. R. 1998. Variação espaço-temporal da meiofauna, ao longo de um período quinzenal, em um ponto fixo da Praia Vermelha, Rio de Janeiro. Publicacão ACIESP 104(4):179-193.

Giere, O. 1993. Meiobenthology - The microscopic fauna in aquatic sediments. Berlin, Springer Verlag. 327p.

Harrigue, A. C.; Gaozza, L.; Montella, A. \& Misic, C. 2006. Benthic communities on a sandy Ligurian beach (NW Mediterranean). Hydrobiologia 571:383-394.

Kaiser, M. J.; Atrill, M. J.; Jennings, S.; Thomas, D. N.; Barnes, D. K. A.; Brierley, A. S.; Polunin, N. V. C.; Rafaelli, D. G. \& Williams, P. J. LE B. 2005. Marine ecology: processes, systems, and impacts. Oxford, Oxford University. 557p.

Kinne, O. 1971. Salinity. In: Kinne, O. ed. Marine ecology: A comprehensive, integrated treatise on life in oceans and coastal waters. New York. Wiley-Interscience. v. 3, p. 342-397.

Kotwicki, L.; Szymelfenig, M.; Throch, M.; Urban-Malinga, B. \& WeslawsKi, J. M. 2005a. Latitudinal biodiversity patterns of meiofauna from sandy littoral beaches. Biodiversity and Conservation 14:461-474

Kotwicki, L.; Troch, M.; Urban-Malinga, B.; Gheskiere, T. \& Weslawski, J. M. 2005b. Horizontal and vertical distribution of meiofauna on sandy beaches of the North sea (The Netherlands, Belgium, France). Helgolander Marine Research 59:255-254.

Krause, G. \& Soares, C. 2004. Analysis of beach morphodynamics on the Bragantinian mangrove peninsula (Pará, North Brazil) as prerequisite for coastal zone management recommendations. Geomorphology 60:225-239.

Martorrano, L. G; Pereira, L. C.; Cézar, E. G. M. \& Pereira, I. C. B. 1993. Estudos climáticos do estado do Pará, classificação climática (Köppen) e deficiência hídrica (Thornthwhite, Mather). Belém, SUDAM / EMBRAPA, SNLCS. 53p.

McLachlan, A. 1980. Intertidal zonation of macrofauna and stratification of meiofauna on high energy sandy beaches in the Eastern Cape, South Africa. Transactions of the Royal Society of South Africa 44(2):213-223.

1983. Sandy beach ecology - a review. In: McLachlan, A. \& Erasmus, T. eds. Sandy beaches as ecossystems. Cape Town, W. Junk, The Haugue. p.321-380.

Mclachlan, A. \& Brown, A. 2006. The ecology of sandy shores. Amsterdam, Academic. 373p.

Mclachlan, A. \& Dorvlo, A. 2005. Global patterns in sandy beach macrobenthic communities. Journal of Coastal Research 21(4):674-687.

McLachlan, A. \& Turner, I. 1994. The interstitial environment of sandy beaches. Marine Ecology 15(3/4):177-211.

McLachlan, A.; Wooldridge, T. \& Dye, A. H. 1981. The ecology of sandy beaches in Southern Africa. South Arican Journal of Zoology 16:219-231.

Medeiros, L. R. A. 1984. Meiofauna da região entremarés do litoral norte do estado de São Paulo. Ciência e Cultura 36(supl.):9-30.

Moodley, L.; Chen, G.; Heip, C. \& Vincx, M. 2000. Vertical distribution of meiofauna in sediments from contrasting sites in the Adriatic Sea: clues to the role of abiotic versus biotic control. Ophelia 53:203-212.

Moraes, B. C.; Costa, J. M. N.; Costa, A. C. L. \& Costa, M. H. 2005. Variação espacial e temporal da precipitação no estado do Pará. Acta Amazonica 35(2):207-214.

Ndaro, S. G. M.; SJoling, S. \& Olaffsson, E. 1995. Small-scale variation in major meiofaunal taxa and sediment chemistry in tropical sediments. Ambio 24:470-474.

Netto, S. A.; Attrill, M. J. \& Warwick, R. M. 1999. The effect of a natural physical disturbance on the structure of meiofauna and macrofauna communities in the intertidal sand-flat of Rocas Atoll (NE, Brazil). Journal of Sea Research 42:291-302.

Nogueira, C. R. \& Skowronski, R. S. P. 2001. Hydrodinamic influence on meiofauna distribution on two sandy beaches in Rio de Janeiro. Tropical Oceanography 29(2):161-174. 
'Olafsson, E. 1991. Intertidal meiofauna of four sandy beaches in Iceland. Ophelia 33(1):55-65.

Paula, J. H. C.; Rosa Filho, J. S.; Souza, A. L. B. \& Aviz, D. E. 2006. A meiofauna como indicadora de impactos da carcinicultura no estuário de Curuçá (PA). Boletim do Labohidro 19:61-72.

Pennak, R. W. 1951. Comparative ecology of interstitial fauna of fresh-water and marine beaches. Animal Biology 27(6):449-480.

Pinto, T. K. O. \& Santos, P. J. P. 2006. Meiofauna community structure variability in a Brazilian tropical sandy beach. Atlântica 28(2):117-127.

Pollock, L. W. 1970. Ecology of intertidal meiobenthos. Smithsonian Contributions to Zoology 76:141-148.

Rafaelli, D. \& Hawkins, S. 1996. Intertidal ecology. London, Chapman \& Hall. 356p.

Rodríguez, J. G. 2004. Community structure of the intertidal meiofauna along a gradient of morphodynamic states on an exposed North Sea beach. Sarsia 89:22-32.

Rodríguez, J. G.; LASTRA, M. \& LÓPEZ, J. 2003. Meiofauna distribution along a gradient of sandy beaches in Northern Spain. Estuarine, Coastal and Shelf Science 58:63-69.

Rosa Filho, J. S.; Paula, J. H. C.; Magalhães, C. M. B. \& Souza, A. L. B. 2007. Variações espaço-temporais na estrutura das associações bentônicas nas adjacências de uma fazenda de cultivo de camarão no estuário de Curuçá (PA). In: Barroso, G. F.; Poersch, L. H. \& Cavalli, R. O. eds. Sistemas de cultivos aqüícolas costeiros no Brasil: recursos, tecnologias e aspectos ambientais e sócio-econômicos. Rio de Janeiro, Museu Nacional. p.303-315.

Short, A. D. 2003. Handbook of beach and shoreface morphodynamics. New York, John Wiley \& Sons. 375p.

Snelglove, P. V. R. \& Buttman, C. A. 1994. Animal-sediment relationships revisited: cause versus effect. Oceanography and Marine Biology: an Annual Review 32:111-177.

Souza-Filho, P. W. M.; Cohen, M. C. L.; Lara, R. J.; Lessa, G. C.; Koch, B. \& Behling, H. 2004. Holocene coastal evolution and facies model of the Bragança macrotidal flat on the Amazon Mangrove Coast, northen Brazil. Journal of Coastal Research SI(39):306-310.

Souza-filho, P. W. M.; Tozzi, H. A. M. \& El-Robrini, M. 2003. Geomorphology, land use and environmental hazards in Ajuruteua macrotidal sand beach, northern Brazil. Journal of Coastal Research SI(35):580-589.

Souza-Santos, L. P.; Ribeiro, V. S. S.; Santos, P. J. P. \& FonsêcaGenevois, V. 2003. Seasonality of intertidal meiofauna on a tropical sandy beach in Tamandaré Bay (Northeast Brazil). Journal of Coastal Research SI(35):369-377.

Thorson, G. 1957. Bottom communities (sublittoral and shallow shelf). Geological Society of America 67:461-534.

Wandeness, A. W.; Esteves, A. M.; Subrinho, S. J. R. P. \& Nogueira, C. S. R. 1997. Meiofauna da zona entre-marés da praia dos Anjos, Arraial do Cabo, RJ. Oecologia Brasiliensis 3:93-106.

Wright, L. D. \& ShorT, A. D. 1984. Morphodynamic variability of surf zones and beaches: A Synthesis. Marine Geology 56:93-118.

Recebido em dezembro de 2006. Aceito em julho de 2008. ISSN 0073-4721

Artigo disponível em: www.scielo.br/isz 